\title{
Salt-cured Atlantic cod skin: a sustainable source of acid-soluble type I collagen
}

\author{
Ezequiel R. Coscueta ${ }^{1, *}$, María Emilia Brassesco ${ }^{1}$ and Manuela Pintado 1,* \\ ${ }^{1}$ Universidade Católica Portuguesa, CBQF - Centro de Biotecnologia e Química Fina - Laboratório \\ Associado, Escola Superior de Biotecnologia, Rua Diogo Botelho 1327, 4169-005 Porto, Portugal; \\ *Correspondence: ecoscueta@porto.ucp.pt, mpintado@porto.ucp.pt
}

\begin{abstract}
Collagen is the most abundant protein in the animal kingdom. Industrial collagen is mainly bovine and porcine origin. However, due to religious beliefs, allergic issues, and infectious diseases, alternative sources of collagen as marine are gaining increasing interest. In this work, the acid-soluble collagen (ASC) were extracted from salt-cured Atlantic cod (Gadus morhua) skin and characterized. The extraction yield was about $2.0 \%$, equivalent to the extraction yield reported for other fish skins. The electrophoretic pattern showed the typical type I structure ( $\alpha, \beta$ and $\gamma$ chains). UV-VIS and FTIR absorbance spectra suggested a very pure ASC with an intact triple helical structure. The integrity and the adequate porosity required for different applications were then confirmed by electron micrograph. Our findings allow us to say that, for the first time, we extracted acid-soluble type I collagen from salt-cured Atlantic cod skin, with characteristics suitable for application in various fields, such as biomedical.
\end{abstract}

Keywords: salt-cured cod skin; Gadus morhua; collagen; fishery by-products; biomaterials

\section{Introduction}

Collagen is a general term for structural proteins present in the extracellular matrices of animals. This is one of the major proteins among the body's total proteins, comprising about $30 \%$ [1]. Generally, collagen is formed by polypeptide chains constituted by repeating triplets Gly-X-Y of Glycine and two other amino acids, where proline and hydroxyproline (Hyp) are the most common, including ca. 1000 total amino acids [2,3]. Collagen polypeptide chains adopt a 3D superhelical structure that provides an ideal geometry for inter-chain hydrogen bonding. The triple helices selfassemble in a staggered formation to form collagen fibrils. Those fibrils are also packed together to form collagen fibres, responsible for the tensile strength of this material [4]. So far, about 29 types of collagen have been recognized as structural and biologically active components in multifarious tissues including skin, bone and cartilage [5].

Type I collagen is the most abundant and is widely used for food, cosmetic, biomedical, and pharmaceutical applications [6]. Due to the dominant presence of hydrophobic amino acids, it exhibits high emulsifying ability for hydrophilic-hydrophobic partitioning. Owing to its unique physical and biological properties, collagen has several applications. In food industry as a food additive to improve the texture, water holding capacity and stability of several food products and in packaging industries for microencapsulation and light-sensitive coatings formation. In biomedical industry for the encapsulation of drugs and in cosmetics for production of creams and gels with high moisturizing, anti-ageing, anti-wrinkle or UV radiation protective actions [7]. In particular, its low immunogenicity and high biocompatibility make collagen a biopolymer of high interest for biomedical applications [8]. Moreover, collagen-derived peptides have numerous other bioactivities such as antimicrobial activity, mineral-binding capacity, lipid-lowering effect, immunomodulatory activity and beneficial effects on the skin, bone, or joint health. 
However, the source from which it is extracted can affect these properties, mainly due to contamination, loss of stability. Besides, the source may also constrain the consumer acceptance due to moral or religious reasons. Collagen is primarily obtained from land animal sources like bovine and porcine origin. In the last years, due to the outbreak of diseases such as Bovine Spongiform Encephalopathy (BSE), Transmissible Spongiform Encephalopathies (TSE), and Foot and Mouth Disease (FMD) among that animals, the search for alternative sources of collagen has increased [2]. In the past two decades, academia and industry have explored marine by-products as a reliable and economic source of collagen. It includes marine fish, starfish, sponges, jellyfish, squid, etc. [1,3,6,9]. The work performed till date proves that marine fish may be a promising source.

Worldwide, Portugal is the 3rd highest fish consuming country globally [10], with a consequent large fishing industry that produces the largest amount of fishery waste. The pattern of fish consumption in Portugal includes not only the most abundant species in national waters but imported salt-cured cod (Gadus morhua), which represents one of the main sources of fish residues in the country. Those residues may account for an average of $60 \%$ of the total fish weight, being $30 \%$ skin and bones. More than $80 \%$ of the cod skin's protein content is collagen [3], which positions it as a source of high-value collagen that is currently wasted or used in animal feed.

Some studies already characterised the collagen and gelatine from the skin of some cod species (Baltic cod [11] and Pacific cod [9,12]); however, nobody has yet performed studies on salt-cured Atlantic cod (Gadus morhua). So, the aim of this study was to extract collagen from discarded saltcured cod skin and demonstrate the feasibility of technology. Besides, for the extracted collagen it was analysed the quality and stability of the extracted collagen to envisage suitable future use in several applications such as food, cosmetic and biomedical.

\section{Results}

\subsection{ASC extraction}

The most used methodology to extract collagen from fish by-products has been the acid extraction [13]. In this process, the collagen is extracted with a dilute weak acid solution (e.g. acetic, lactic), and the product obtained is usually called acid-soluble collagen (ASC) [4]. We were able to extract ASC from the salt-cured cod skin with a yield ca. $2.0 \%$ based on the dry weight.

\subsection{ASC electrophoretic pattern}

The ASC was analysed by Tricine-SDS-PAGE (Figure 1). Lane 2 shows the electrophoretic pattern of the cod skin ASC, with the presence of the typical subunits of type I collagen $(\alpha 1, \alpha 2, \beta$ and $\gamma$ ). For $\alpha 1$ and $\alpha 2$, the observed molecular masses were $135 \mathrm{kDa}$ and $125 \mathrm{kDa}$, respectively, while for the $\beta$ chain (dimers) and $\gamma$ chain (trimers) higher than $200 \mathrm{kDa}$. The existence of those last two high molecular mass components indicates that cod skin ASC contains inter- and intra-molecular crosslinked proteins. The observed pattern was like the calf skin type I collagen (lane 1). 


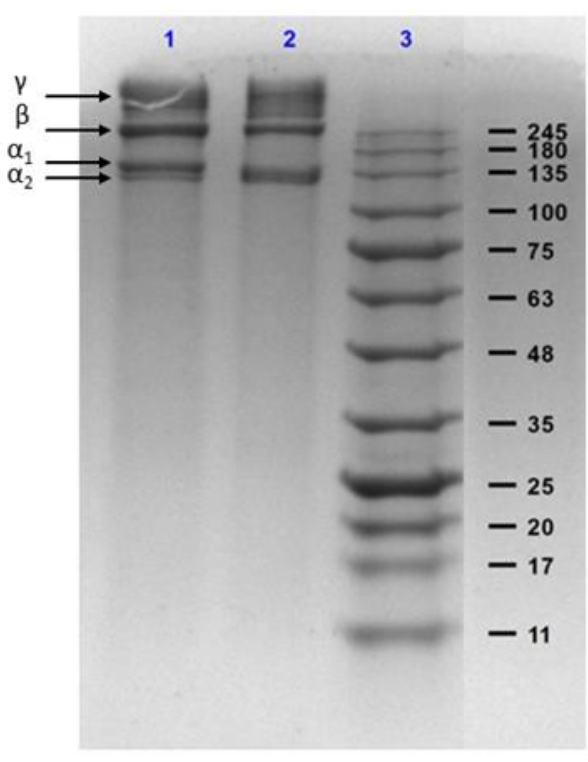

Figure 1. SDS-PAGE: Calf skin type I collagen (lane 1), collagen purified from cod skin by acid methodology (lane 2); molecular mass marker in kDa (lane 3).

\subsection{UV-VIS spectra}

The UV-VIS absorbance data of cod skin ASC and calf skin type I collagen are presented in Figure 2. The cod skin ASC exhibited a maximum absorption at $213 \mathrm{~nm}$, which was closer to calf skin type I collagen $(229 \mathrm{~nm})$. Both spectra also showed a subtle peak around $280 \mathrm{~nm}$.

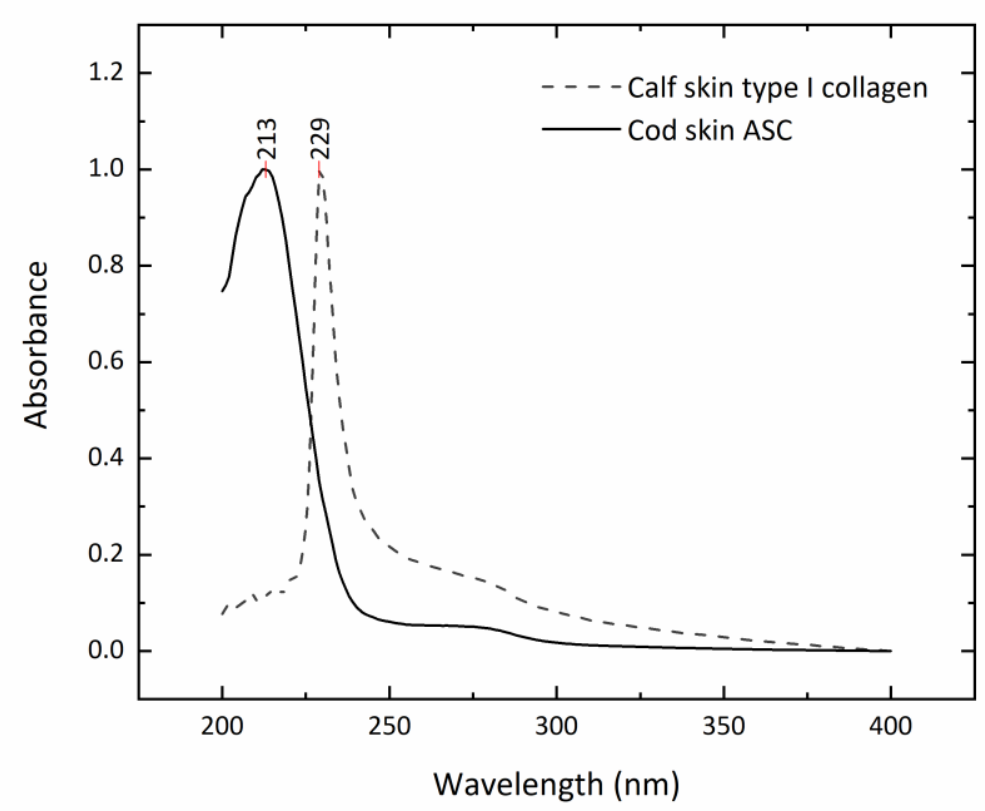

Figure 2. UV-VIS spectra of calf skin type I collagen and cod skin ASC.

\subsection{FTIR spectra}

Figure 3 shows the cod skin ASC FTIR spectra. The amide A band of cod skin ASC was observed at $3297 \mathrm{~cm}^{-1}$, which corresponds to $\mathrm{N}-\mathrm{H}$ stretching vibration. The amide $B$ band is usually close to 3080 $\mathrm{cm}^{-1}$ and is associated with an asymmetrical $\mathrm{CH}_{2}$ stretch. In cod skin ASC, the amide $B$ band was at $3077 \mathrm{~cm}^{-1}$. The amide I, II, and III bands of cod skin ASC were at 1645, 1548, and $1238 \mathrm{~cm}^{-1}$, respectively. 
The amide I, II, III bands correspond to the molecular-order degree of collagen [14]. Moreover, another important parameter to determine for collagen is the absorption area ratio between the amide III band and the $\mathrm{CH}_{2}$ bending band at $1450-1454 \mathrm{~cm}^{-1}$. The ratio for cod skin ASC was 1.083 .

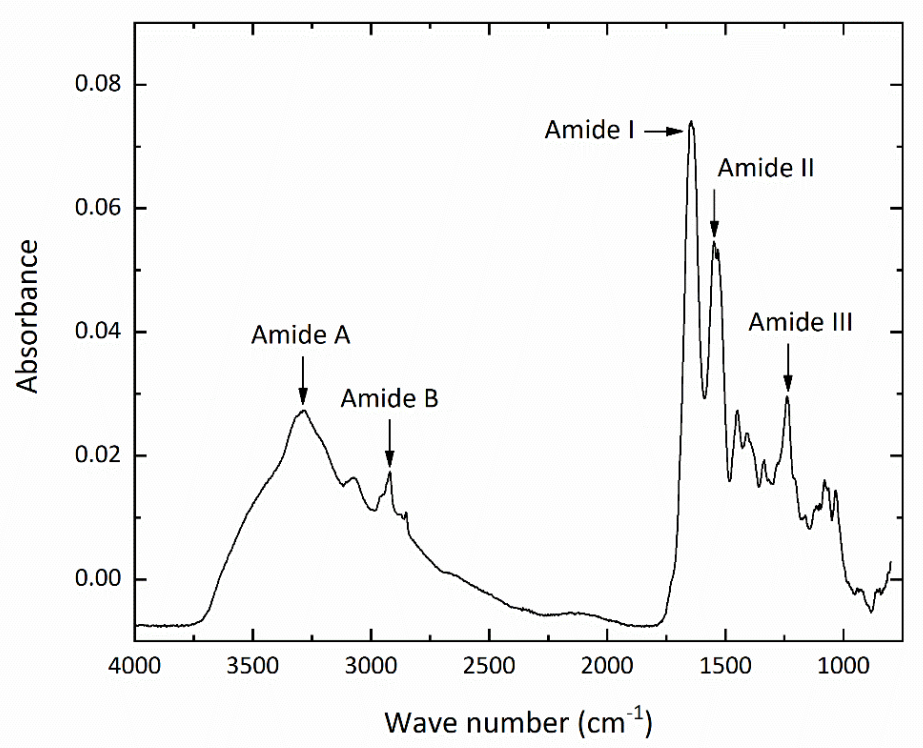

Figure 3. FTIR spectra of cod skin ASC.

\subsection{SEM analysis}

Figure 4 shows the electron micrographs of the lyophilized cod skin ASC and calf skin type I collagen. The cod skin ASC exhibited random windings of coil-like structures, with a porous and three-dimension interconnected fibre structure like calf skin type I collagen (Figures 4.B and 4.D). In the case of the cod skin ASC, we saw a less compact random winding structure concerning calf skin type I collagen, which could indicate a greater porosity. 

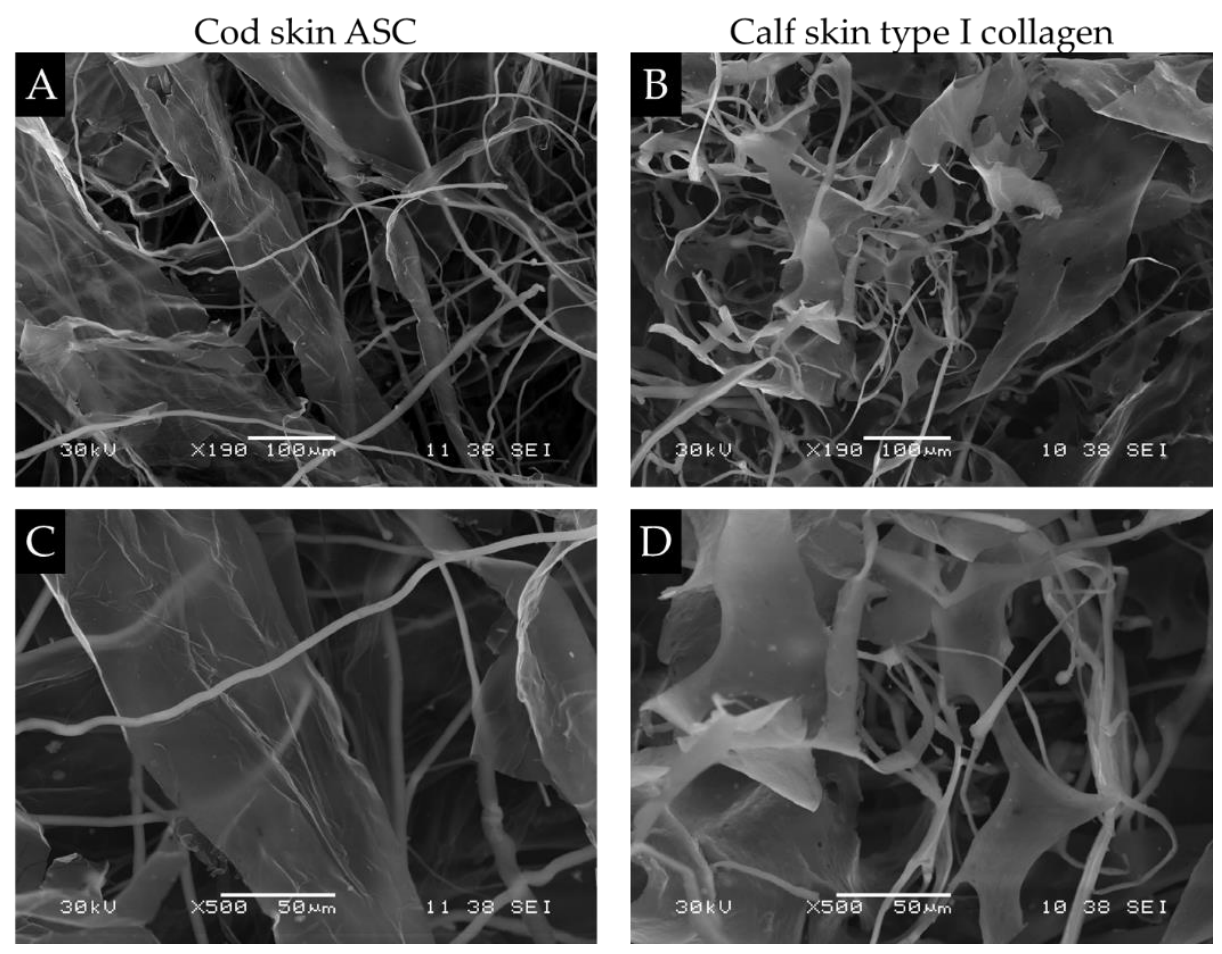

Figure 4. SEM images: A) cod skin ASC, x190 magnification; B) calf skin type I collagen, x190 magnification; C) cod skin ASC, x500 magnification; D) calf skin type I collagen, x500 magnification.

\section{Discussion}

The performance of the extraction is consistent with what has been reported so far. Sionkowska et al. [15] and Arumugam et al. [16] reported lower yields, 1.5\% (from Brama australis skin) and 1.9\% (from Sole fish skin), respectively. On the other hand, similar and higher yields have been reported for cuttlefish and balloon fish, 2.0\% [17] and 4.0\% [2], respectively. Furthermore, Sousa et al. [18] obtained about $5.72 \%$ from the same species of cod (Gadus morhua) here studied, but not from the skin but the swim bladders. The ASC provides an idea of the amount of collagen that could be present in the skin or body part of a particular specie; however, it is not a quantitative extraction of collagen, since there is evidence that part of the collagen cannot be extracted using this simple procedure [18]. This approach is widely used since the methodology can be easily applied for industrial extraction of collagen. However, despite presenting relevant yields, this value is still low, when we know that $80 \%$ of the protein content of fish skin is collagen. It is important to highlight that in all the mentioned studies no one used salt-cured fish, just fresh material; so this is the first report for fish salt-cured material, with the main challenge being the effect of the drying process with a high amount of salt since the collagen is a very unstable molecule.

The observed electrophoretic pattern was similar not only to the calf skin type I collagen, but to the Atlantic cod swim bladders collagen [18], and the skin collagen of most other fish species [1,2].

Regarding its UV-VIS characterization, a well-established characteristic of triple-helical collagen is a maximum absorbance between $210-240 \mathrm{~nm}$. Wang et al. showed a maximum absorption peak at $218 \mathrm{~nm}$ for the loach skin ASC [1]. Similarly, Pacific cod skin and channel catfish skin ASCs exhibited maximums at $231 \mathrm{~nm}$ [9] and $232 \mathrm{~nm}$ [19], respectively. The UV-VIS absorption is possibly related to $-\mathrm{COOH}, \mathrm{CONH}_{2}$ groups in polypeptide chains and $\mathrm{n} \rightarrow \pi^{*}$ transition of $\mathrm{C}=\mathrm{O}$ in peptide bond [20]. Besides, as Figure 2 shows, low absorbance was obtained at $280 \mathrm{~nm}$, due to the low presence of aromatic amino acids in ASC, which is an evidence of the high purity of the ASC (low presence of non-collagen proteins). Similar findings were reported in collagen from eel-fish [21], loach skin [1] and balloon fish skin [2]. 
In the FTIR spectra we observed five typical amide bands similar to other type-I collagens [1]. Hukmi et al. showed that the amide A peak of ASC of silver catfish skin collagen is at $3457.07 \mathrm{~cm}^{-1}$ [22]. Arumugam et al. reported that the $\mathrm{N}-\mathrm{H}$ stretching vibration of sole skin fish is shifted to 3310.21 $\mathrm{cm}^{-1}$ [16]. According to Doyle [23], N-H stretching vibration ranges from 3400 to $3440 \mathrm{~cm}^{-1}$ and when the $\mathrm{NH}$ group of a peptide is associated with a hydrogen bond the peak position is shifted to a lower frequency. Our result revealed that the NH groups of cod skin ASC formed hydrogen bonds with carbonyl groups present in the peptide chain. In comparison, Sionkowska et al. reported the amide $B$ band of Brama australis collagen at $3079 \mathrm{~cm}^{-1}$ [15]. Similar results showed the amide $B$ bands of loach skin ASC at $2928 \mathrm{~cm}^{-1}$ [1] and for turbot skin ASC at $2913 \mathrm{~cm}^{-1}$ [24]. The amide I, II, and III bands of cod skin ASC were also in agreement with the findings of Sun et al. [9], who studied the Pacific cod skin ASC and reported the corresponding wavelengths at $1655.52 \mathrm{~cm}^{-1}, 1540.21 \mathrm{~cm}^{-1}, 1232.74 \mathrm{~cm}^{-1}$. The absorption area ratio between the amide III band and the $\mathrm{CH}_{2}$ bending band at $1450-1454 \mathrm{~cm}^{-1}$ may reflect the stability of a triple helical structure, being stable at a ratio close to 1.0 [25]. The ratio for cod skin ASC (1.083) was close to 1.0, indicating the intact triple helical structure in the obtained product. Similar findings were reported for other ASCs: Pacific cod skin [9]; turbot skin [24]; Spanish mackerel skin [26]; and loach skin [1]. We consider important to highlight this integrity because it means that even under the condition of curing and salting the collagen was preserved in the native structure.

Typically, random windings of coil-like structures are characteristic of the fibrous nature of the collagen [16]. As seen in the electron micrographs, the cod skin ASC exhibited this kind of morphology, not only calf skin type I collagen but acid-soluble collagen from Pacific cod bone [27]. Other authors reported similar structures for the silver catfish skin [22], sole fish skin [16] and Pacific cod bone [27] with the typical structure of type I ASC (flaky and porous). We related the lace-like fibres to the native helical structure of collagen, which reinforces our FTIR results. The microstructure patterns (pore size, porosity, interconnectivity and surface area) is widely recognized as important parameters for a biomaterial to understand its biomedical importance. The space between the interlaced sheets represents the porosity of the collagen, which facilitates the incorporation of any compound, such as a drug [16]. The greater porosity we saw may indicate greater incorporation capacity per mass unit. At the same time, this can make the cod skin ASC material more fragile than calf skin type I collagen. This may be related to the lower imino acid content (Hyp) of cold-water fish collagen [28]. The Hyp plays a very important role in stabilizing the triple helix. That is why the collagens of mammals and even warm-water fish are more resistant and more thermally stable, as they have more compact and stable helical structures due to their higher Hyp content [29].

\section{Materials and Methods}

\subsection{Materials and cod samples}

Calf skin type-I collagen was purchased from Sigma-Aldrich (St. Louis, MO, USA) and molecular weight marker was purchased from NZYTech, Lisboa, Portugal. All other reagents used were of analytical grade.

Salt-cured cod skin from Atlantic cod (Gadus morhua) was kindly provided by Pascoal \& Filhos S.A. The skin was obtained as by-product before the cutting and processing of fish meat to obtain different portions to sell or for ready to eat meals. The samples were transported at room temperature and stored at $-20^{\circ} \mathrm{C}$, before processing.

\subsection{Pre-treatment of skin}

The pre-treatment of skin was carried out following the methodology of Arumugam et al. [16], with some modifications. First, the cod skins were washed with tap water 3 times at $4{ }^{\circ} \mathrm{C}$ to remove the salt, residual fat and flesh of fish (by the action of high salt concentration) and then cut into small pieces. Then, those pieces were mixed with $0.1 \mathrm{M} \mathrm{NaOH}$ at $4{ }^{\circ} \mathrm{C}$ to remove non-collagenous proteins and pigments at a sample-to-solution ratio of $1: 10(\mathrm{w} / \mathrm{v})$, about $4 \mathrm{~h}$. The mixture was centrifuged (Hettich Universal 320R) at $3857 \mathrm{RCF}$ for $15 \mathrm{~min}$ at $4{ }^{\circ} \mathrm{C}$. The resulting solid residue was washed with 
distilled water 3 times until neutral $(\mathrm{pH} 7)$ at $4{ }^{\circ} \mathrm{C}$ and then centrifuged according to the conditions described above.

\subsection{Extraction of acid-soluble collagen (ASC)}

The extraction of ASC was carried out following the methodology of Arumugam et al. [16], with some modifications. After the pre-treatment, the solid residue was soaked in $0.5 \mathrm{M}$ acetic acid with solid (initially weighed)/solvent ratio of $1: 30(\mathrm{w} / \mathrm{v})$ at $4{ }^{\circ} \mathrm{C}$. After $24 \mathrm{~h}$, the mashed was centrifuged at $3857 \mathrm{RCF}$ for $15 \mathrm{~min}$ at $4{ }^{\circ} \mathrm{C}$, and the obtained supernatant was salted-out by the addition of ground $\mathrm{NaCl}$ to a final concentration of $0.9 \mathrm{M}$. The mixture was left overnight and centrifuged at $3857 \mathrm{RCF}$ for $30 \mathrm{~min}$ at $4{ }^{\circ} \mathrm{C}$. The resulting precipitate was dissolved in $0.5 \mathrm{M}$ acetic acid and dialysed (Spectra/Por 4 Dialysis Membrane MWCO 12-14 kD, Spectrum Laboratories Inc.): against $0.1 \mathrm{M}$ acetic acid the first $24 \mathrm{~h}$; against deionised water the subsequent $48 \mathrm{~h}$. The dialysate was renewed every 8 h. Finally, the purified acid-soluble collagen (ASC) was lyophilized, sealed in polythene bags and stored at $-20^{\circ} \mathrm{C}$ for subsequent analysis.

\subsection{Tricine-SDS-PAGE}

Tricine-SDS-PAGE [[30]] was prepared using $4 \%$ stacking and $10 \%$ resolving gel. $1.0 \mathrm{mg} / \mathrm{mL}$ cod skin ASC and calf skin type I collagen (calf skin ASC) were mixed in $0.5 \mathrm{M}$ acetic acid, diluted $1 / 2$ with sample buffer, incubated at $80{ }^{\circ} \mathrm{C}$ for $20 \mathrm{~min}$, and centrifuged at $21382 \mathrm{RCF}$ for $10 \mathrm{~min}$. Twenty microliters of supernatants were loaded in gel. The electrophoresis ran at a constant voltage of $75 \mathrm{~V}$ for about 5 min followed by $150 \mathrm{~V}$. After electrophoresis, the gel was stained in Coomassie blue G$250(0.25 \%, \mathrm{w} / \mathrm{v})$, staining solution for $1 \mathrm{~h}$ and discoloured overnight. The final image was acquired with the ChemiDoc ${ }^{\mathrm{TM}}$ XRS+ and analysed by the Imaging System Image Lab ${ }^{\mathrm{TM}}$ Software Version 6.0.1.34.

\subsection{UV-VIS measurement}

The ASCs (cod skin and calf skin) were dissolved in $0.5 \mathrm{M}$ acetic acid to a concentration of 1.0 $\mathrm{mg} / \mathrm{mL}$ and centrifuged at $4146 \mathrm{RCF}$ for $10 \mathrm{~min}$ at $4{ }^{\circ} \mathrm{C}$. The spectra were recorded using a microplate reader (Multiskan GO, Thermo Scientific) in the range of 200-400 nm.

\subsection{ATR-FTIR analysis}

Lyophilised cod skin ASC FTIR spectra were acquired at an average of 30 measurements and a resolution of $4 \mathrm{~cm}^{-1}$ from 4000 to $400 \mathrm{~cm}^{-1}$ and were expressed as normalized absorbance according to the maximum of each spectrum. The used infrared spectrometer was ABB MB3000 (ABB, Switzerland), equipped with a deuterated triglycine sulphate detector and provided with a horizontal reflection accessory MIRacleTM (PIKE Technologies, USA), for attenuated total reflectance (ATR), with a diamond crystal plate/Se.

\subsection{Scanning Electron Microscopy (SEM)}

The ASC morphology was evaluated by SEM using JSM-5600 Lv Microscope (JEOL, Tokyo, Japan). The samples were coated with gold/palladium using a Sputter Coater (Polaron, Bad Schwalbach, Germany). SEM was operated at low vacuum mode at a voltage of $30 \mathrm{kV}$.

\section{Conclusions}

We obtained for the first-time acid-soluble collagen from salt-cured Atlantic cod with an extraction yield about $2.0 \%$. The obtained ASC consisted mainly of type I collagen with the typical subunits: two different $\alpha$ chains; $\beta$ chains (dimers) and $\gamma$ chains (trimers). The UV-VIS absorbance spectra suggested a high purity product with the typical triple-helical pattern, which was confirmed by FTIR and SEM. In short, it was possible to extract type I collagen with high purity from a new fish source, which is one of the largest fishery wastes in Portugal. However, the weak point of our study, 
as well as the majority of those carried out so far, is the low extractive yield, and there is still a lot of material to recover, that is, a lot of lost value. This leaves the doors open to the need to optimize process variables as well as develop new, more efficient processes.

As the last assessment, considering the wide range of applications that have been recognized for collagen, as well as the many others that would be unveiled in the future, this work makes an important contribution by providing an alternative and sustainable source of such material.

Author Contributions: Conceptualization, E.R.C. and M.P.; methodology, E.R.C.; software, E.R.C. and M.E.B.; validation, E.R.C. and M.E.B.; investigation, E.R.C. and M.E.B.; resources, M.P.; writing-original draft preparation, E.R.C. and M.E.B.; writing-review and editing, E.R.C., M.E.B. and M.P.; supervision, M.P.; project administration, M.P.; funding acquisition, M.P. All authors have read and agreed to the published version of the manuscript.

Funding: This research was funded by Foundation for Science and Technology (FCT) through the "MultiBiorefinery" Project (POCI-01-0145-FEDER-016403).

Conflicts of Interest: The authors declare no conflict of interest.

\section{References}

1. Wang J, Pei X, Liu H, Zhou D. Extraction and characterization of acid-soluble and pepsin-soluble collagen from skin of loach (Misgurnus anguillicaudatus). Int J Biol Macromol. 2018;106:544-50 DOI: 10.1016/j.ijbiomac.2017.08.046.

2. Huang YR, Shiau CY, Chen HH, Huang BC. Isolation and characterization of acid and pepsinsolubilized collagens from the skin of balloon fish (Diodon holocanthus). Food Hydrocoll. 2011;25(6):150713 DOI: 10.1016/j.foodhyd.2011.02.011.

3. Sotelo CG, Comesaña MB, Ariza PR, Pérez-Martín RI. Characterization of Collagen from Different Discarded Fish Species of the West Coast of the Iberian Peninsula. J Aquat Food Prod Technol. 2016;25(3):388-99 DOI: 10.1080/10498850.2013.865283.

4. Silva TH, Moreira-Silva J, Marques ALP, Domingues A, Bayon Y, Reis RL. Marine origin collagens and its potential applications. Mar Drugs. 2014;12(12):5881-901 DOI: 10.3390/md12125881.

5. Li LY, Zhao YQ, He Y, Chi CF, Wang B. Physicochemical and antioxidant properties of acid- And pepsin-soluble collagens from the scales of Miiuy croaker (Miichthys miiuy). Mar Drugs. 2018;16(10) DOI: 10.3390/md16100394.

6. Kittiphattanabawon P, Benjakul S, Visessanguan W. Characterisation of acid-soluble collagen from skin and bone of bigeye snapper ( Priacanthus tayenus ). Food Chem. 2005;89:363-72 DOI: 10.1016/j.foodchem.2004.02.042.

7. Atef M, Mahdi Ojagh S. Health benefits and food applications of bioactive compounds from fish byproducts: A review. J Funct Foods. 2017;35:673-81 DOI: 10.1016/j.jff.2017.06.034.

8. Gu L, Shan T, Ma Y xuan, Tay FR, Niu L. Novel Biomedical Applications of Crosslinked Collagen. Trends Biotechnol. 2019;37(5):464-91 DOI: 10.1016/j.tibtech.2018.10.007.

9. Sun L, Li B, Song W, Si L, Hou H. Characterization of Pacific cod (Gadus macrocephalus) skin collagen and fabrication of collagen sponge as a good biocompatible biomedical material. Process Biochem. 2017;63(August):229-35 DOI: 10.1016/j.procbio.2017.08.003.

10. Madsen AO, Chkoniya V. Fish Consumption in the Age of the Information Society-The Evolution of the Fish Sector in Portugal. Eur J Soc Sci. 2019;8632(August):36-50.

11. Sadowska M, Ko I, Niecikowska C. Isolation of collagen from the skins of Baltic cod ( Gadus morhua ). Food Chem. 2003;81:257-62.

12. Chen T, Hou H. Protective effect of gelatin polypeptides from Paci fi c cod ( Gadus macrocephalus ) against UV irradiation-induced damages by inhibiting in $\mathrm{fl}$ ammation and improving transforming growth factor- $\beta$ / Smad signaling pathway. JPB. 2016;162:633-40 DOI: 10.1016/j.jphotobiol.2016.07.038.

13. Karim AA, Bhat R. Fish gelatin: properties, challenges, and prospects as an alternative to mammalian gelatins. Food Hydrocoll. 2009;23(3):563-76 DOI: 10.1016/j.foodhyd.2008.07.002.

14. J.H. Muyonga, C.G.B. Cole KGD. Fourier transform infrared ( FTIR ) spectroscopic study of acid soluble collagen and gelatin from skins and bones of young and adult Nile perch ( Lates niloticus ). Food Chem. 2004;86:325-32 DOI: 10.1016/j.foodchem.2003.09.038. 
15. Sionkowska A, Kozłowska J, Skorupska M, Michalska M. Isolation and characterization of collagen from the skin of Brama australis. Int J Biol Macromol. 2015;80:605-9 DOI: 10.1016/j.ijbiomac.2015.07.032.

16. Arumugam GKS, Sharma D, Balakrishnan RM, Ettiyappan JBP. Extraction, optimization and characterization of collagen from sole fish skin. Sustain Chem Pharm. 2018;9:19-26 DOI: 10.1016/j.scp.2018.04.003.

17. Nagai T, Yamashita E, Taniguchi K, Kanamori N, Suzuki N. Isolation and characterisation of collagen from the outer skin waste material of cuttlefish (Sepia lycidas). Food Chem. 2001;72(4):425-9 DOI: 10.1016/S0308-8146(00)00249-1.

18. Sousa RO, Alves AL, Carvalho DN, Martins E, Silva TH, Reis RL, et al. Acid and enzymatic extraction of collagen from Atlantic cod ( Gadus Morhua ) swim bladders envisaging health-related applications. J Biomater Sci Polym Ed. 2020;31(1):20-37 DOI: 10.1080/09205063.2019.1669313.

19. Liu H, Li D, Guo S. Studies on collagen from the skin of channel catfish ( Ictalurus punctaus ). Food Chem. 2007;101:621-5 DOI: 10.1016/j.foodchem.2006.01.059.

20. H.G.M. Edwards, D.W. Farwell, J.M. Holder EEL. Fourier-transform Raman spectroscopy of ivory : II . Spectroscopic analysis and assignments. Mol Struct. 1997;435:49-58.

21. Veeruraj A, Arumugam M, Balasubramanian T. Isolation and characterization of thermostable collagen from the marine eel-fish (Evenchelys macrura). Process Biochem. 2013;48(10):1592-602 DOI: 10.1016/j.procbio.2013.07.011.

22. Hukmi N and NS. Isolation and characterization of acid soluble collagen ( ASC ) and pepsin soluble collagen ( PSC ) extracted from silver catfish ( Pangasius sp .) skin. Int Food Res J. 2018;25(December):26017.

23. Doyle BB, Chemistry B. Infrared Spectroscopy of Collagen and Collagen-Like Polypeptides. Biopolymers. 1975;14.

24. Sun J, Zhang J, Zhao D, Xue C, Liu Z, Mao X. Characterization of Turbot (Scophthalmus maximus) Skin and the Extracted Acid-Soluble Collagen. J Ocean Univ China. 2019;18(3):687-92 DOI: 10.1007/s11802019-3837-2.

25. Plepis AM de G, Goissis G, Das-gupta DK. Dielectric and pyroelectric characterization of anionic and native collagen. Polym Eng Sci. 1996;3(24):2932-8.

26. Li Z, Wang B, Chi C, Zhang Q, Gong Y, Tang J, et al. Isolation and characterization of acid soluble collagens and pepsin soluble collagens from the skin and bone of Spanish mackerel ( Scomberomorous niphonius ). Food Hydrocoll. 2013;31(1):103-13 DOI: 10.1016/j.foodhyd.2012.10.001.

27. Wang S, Hou H, Hou J, Tao Y, Lu Y, Yang X, et al. Characterization of acid-soluble collagen from bone of pacific cod (gadus macrocephalus). J Aquat Food Prod Technol. 2013;22(4):407-20 DOI: 10.1080/10498850.2011.654382.

28. Bae I, Osatomi K, Yoshida A, Osako K, Yamaguchi A, Hara K. Biochemical properties of acid-soluble collagens extracted from the skins of underutilised fishes. Food Chem. 2008;108(1):49-54 DOI: 10.1016/j.foodchem.2007.10.039.

29. Berisio R, Granata V, Vitagliano L, Zagari A, Biologica C, Uni V, et al. Imino Acids and Collagen Triple Helix Stability: Characterization of Collagen-like Polypeptides Containing Hyp-Hyp-Gly Sequence Repeats. J Am Chem Soc. 2004;126(37):11402-3 DOI: 10.1021/ja047069h.

30. Gels, T., H. Schagger and G von J. Tricine-sodium dodecyl sulfatepolyacrylamide gel electrophoresis for the separation of proteins in the range from 1 to $100 \mathrm{kDa}$. Anal Biochem. 1987;166:368-379. 